\title{
Planar Resonant Blazed Gratings From a Circuit Model Standpoint
}

\author{
Carlos Molero $^{\circledR}$, Raúl Rodríguez-Berral ${ }^{\circledR}$, Francisco Mesa ${ }^{\circledR}$, Fellow, IEEE, Francisco Medina ${ }^{\circledR}$, Fellow, IEEE, \\ Mohammad Memarian ${ }^{\circledR}$, Senior Member, IEEE, and Tatsuo Itoh ${ }^{\circledR}$, Life Fellow, IEEE
}

\begin{abstract}
The equivalent circuit approach (ECA) is used in this work to analyze and design a previously proposed onedimensional planar blazed grating of the resonant type. The analysis covers both the classical Littrow configuration, when the direction of the relevant diffracted order coincides with that of the incident wave (Bragg blazing), and when these directions are different (off-Bragg blazing). Once the scattering problem of the grating structure is posed as a discontinuity problem inside an equivalent generalized waveguide (corresponding to the unit cell of the original structure) and studied in terms of its equivalent circuit network, the possibility of transferring all the power of the incident plane wave into one single-diffraction order is seen as a simple impedance matching problem. An associated resonance phenomenon is also found to be implicitly associated with this matching condition. This simplifying and fruitful standpoint makes it possible to set up a systematic design procedure to find the specifications of the planar grating for either Bragg or nonBragg blazing operation. Dielectric and strong skin-effect ohmic losses are easily implemented in the ECA and its effects in the practical design of structures are discussed.
\end{abstract}

Index Terms-Bragg gratings, equivalent circuits, metasurfaces, periodic structures.

\section{INTRODUCTION}

B LAZED gratings are a class of planar gratings that can diffract the incident wave into a given diffraction order with great efficiency. The so-called Littrow configuration diffracts back the incident wave into the same direction as

Manuscript received April 18, 2019; revised October 18, 2019; accepted October 29, 2019. Date of publication January 7, 2020; date of current version April 7, 2020. This work was supported in part by the Spanish Agencia Estatal de Investigacin (AEI), Ministry of Science, Innovation and Universities through the European Union FEDER funds under Project TEC2017-84724-P and in part by the Spanish Junta de Andalucía under Grant TIC-112. (Corresponding author: Francisco Medina.)

Carlos Molero is with the Institut National des Sciences Appliquées (INSA) de Rennes, 35708 Rennes, France (e-mail: carlos.molero-jimenez@ insa-rennes.fr).

Raúl Rodríguez-Berral and Francisco Mesa are with the Department of Applied Physics 1, ETS de Ingeniería Informática, University of Seville, 41012 Seville, Spain (e-mail: rrberral@us.es; mesa@us.es).

Francisco Medina is with the Department of Electronics and Electromagnetism, Faculty of Physics, University of Seville, 41012 Seville, Spain (e-mail: medina@us.es).

Mohammad Memarian is with the Department of Electrical Engineering, Sharif University of Technology, Tehran 1458889694, Iran (e-mail: mmemarian@sharif.edu).

Tatsuo Itoh is with the Department of Electrical Engineering, University of California at Los Angeles (UCLA), Los Angeles, CA 90095 USA (e-mail: itoh@ee.ucla.edu).

Color versions of one or more of the figures in this article are available online at http://ieeexplore.ieee.org.

Digital Object Identifier 10.1109/TAP.2019.2963198 the incident wave [1]. Ideally, all the power carried by the impinging plane wave is transferred to a single-diffracted highorder harmonic (usually the $n=-1$ order), thus totally suppressing the specular reflection ( $n=0$ reflected harmonic) and giving rise to complete backscattering of an obliquely incident wave (when the Bragg condition is satisfied, the angles corresponding to the $n=0$ and $n=-1$ Floquet-harmonic are identical). Classical implementations of blazed gratings operating in the optical or microwave ranges have usually made use of corrugated metallic surfaces; in particular, sawtooth, rectangular or even arbitrarily shaped periodic corrugations are considered in the literature [2]-[5].

Nonetheless the classical nature of this problem, the study of blazed gratings continues attracting the attention of the microwave and optics communities [6]-[8]. Very recently, a new research direction has been initiated by developing novel planar or quasi-planar versions of the conventional 3-D thick blazed gratings (note that decreasing the thickness of these structures is especially important in the microwave region of the spectrum to achieve low-profile compact structures). Actually, this research on planar blazed gratings could be seen as a particular manifestation of a general research trend focused on the use of graded metasurfaces and metagratings to achieve almost perfect control of the reflection of plane waves (or beams) by flat surfaces; see, for instance [9]-[15] among many other articles. Some of the authors of this article have already contributed to this topic [16]-[19] reporting metal-dielectric planar metasurfaces that can be considered the equivalent planar versions of the sawtooth grating. The metasurface proposed in [17], fabricated with a standard printed technology process, is shown to reflect almost all the power back into the incidence direction. Thanks to its planar nature, it was used as a side wall of a rectangular waveguide to create a high rejection stopband. An electrically thin structure with a similar behavior can also be found as one of the examples considered in [16]. More recently, an extremely simple planar device leading to blazing operation above a certain threshold angle of incidence has been proposed and studied in [18] and [19]. This simple structure consists of a 1-D periodic array of long transversely resonant strips etched on a conductor backed thin dielectric substrate. The proper choice of the period of the structure makes the angle of the $n=-1$ diffraction order be aimed exactly at the incidence direction. The width of the strips can then be adjusted to ensure perfect backscattering; i.e., zero specular reflection [18], [19]. 
The accurate determination of the optimal metal strip width typically requires the use of a full-wave solver. However, it has been known for a long time [20]-[22] that this class of structures can also be very efficiently analyzed and understood by means of equivalent circuit models. In this research line, some of the authors of this article have contributed to highly accurate analytical circuit models to deal with the scattering of plane waves by arrays of metal strips printed on multilayered substrates [23]. The presence of a conductor ground plane, such as the one existing in the grating analyzed in [18], can easily be incorporated to these models.

The purpose of this article is then to apply the equivalent circuit approach (ECA) reported in [23] to solve the problem originally posed in [18]. Some preliminary results valid for blazed grating in Littrow configuration, also called Bragg blazing, were already reported in a conference paper [24]. In the frame of the ECA, the perfect blazing condition can readily be expressed in terms of the standard perfect matching condition for an appropriate transmission-line circuit. Since all the parameters of the equivalent circuit are obtained in closed form [23], the determination of the required strip width turns into a trivial task. It is worth mentioning that, since the substrate thickness is relatively small in this problem, the role of the first and second high-order Floquet harmonics reflected by the ground plane is essential. Fortunately, this aspect is accurately taken into account by the ECA in [23], in contrast with other circuit models reported in the literature which only considers the reflection of the fundamental harmonic and ignores the influence of the ground plane on the behavior of high-order harmonics. In this way, the proposed circuit model not only provides a satisfactory explanation of the autocollimation process exhibited by the conductor-backed array of metal strips but also provides a systematic, efficient, and extremely fast method to design this kind of gratings. Remarkably, not only on-Bragg blazing (already partially considered in [24]) but also the here-denoted off-Bragg blazing (the direction of the relevant diffracted order does not coincide with the incident one) is conveniently accounted for by the use of this analytical ECA in this article. The possibility of having perfect blazing also for TE-polarized impinging waves is studied in this article as well as the impact of the dielectric and ohmic (metal) losses on the expected response of the system.

This article is structured in nine sections. Section II introduces the basics of the circuit model used in this article. Section III studies the simplest solution that leads to Bragg blazing operation and Section IV describes a systematic design procedure for this class of blazed gratings. The generalization of the resonance (blazing) condition is considered in Section V. In Section VI, the possibility of achieving blazing for TE polarization incidence is discussed. The modeling of off-Bragg blazing is reported in Section VII. The effect of material losses is studied in Section VIII and, finally, a summary of concluding remarks is presented in Section IX.

\section{EQUIVALENT CIRCUIT APPROACH}

The scattering problem under consideration is schematically shown in Fig. 1. A time-harmonic transverse magnetic (TM)

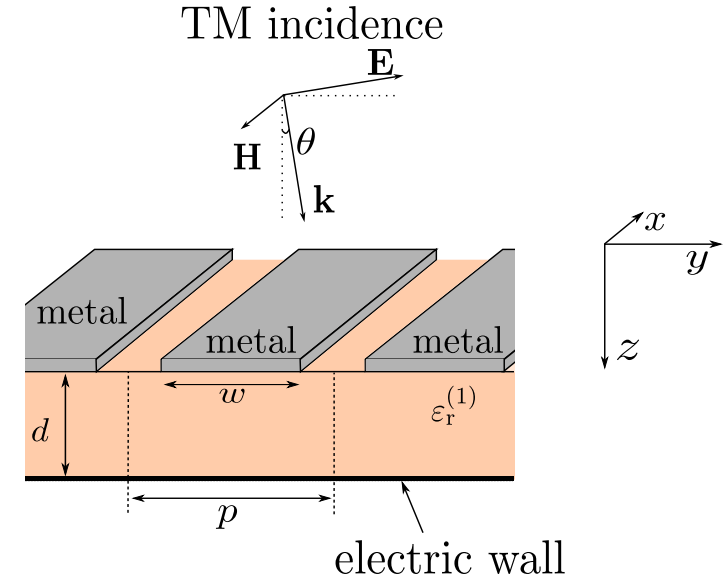

Fig. 1. Sketch of the 1-D diffraction grating analyzed in this article. A periodic distribution (period $p$ ) of metal strips (width $w$ ) is printed on a grounded dielectric slab with relative permittivity $\varepsilon_{\mathrm{r}}^{(1)}$ and thickness $d$. The angle of incidence $(\theta)$ and polarization (TM in this case) are also shown.

plane wave impinges obliquely with an angle $\theta$ on a 1-D periodic metal strip array printed on a grounded dielectric slab. As shown in Fig. 1, the structure is periodic along the $y$-direction (with period $p$ ) and invariant along the $x$-direction. The thickness of the metal strips, which will be considered perfect electric conductors in principle, is neglected. As is well known, if the wavelength $(\lambda)$ of the impinging plane wave fulfills the condition $\lambda>p(1+\sin \theta)$, the incident wave experiences full specular reflection due to the presence of the ground plane and absence of grating lobes. However, for frequencies beyond the onset of the grating lobes regime, the reflected power is split among the specularly reflected (SR), harmonic (order $n=0$ ) and the diffracted ones. In this article we are interested in the control of the blazing phenomenon; namely, the efficient power transfer from the incident wave to a particular diffraction order at a certain frequency (henceforth called blazed frequency). In our study cases in this article, such a particular diffraction order will correspond to the harmonic of order $n=-1$.

A simple equivalent circuit model for this type of gratings was introduced in [23]. Thanks to this model, the conditions that lead to perfect blazing can be posed in terms of an easy impedance-matching problem, which will greatly simplify the elaboration of guidelines for the design of perfect Bragg and off-Bragg blazing structures. For the sake of completeness, the topology of the circuit model and the expressions of its different parameters are here briefly outlined; more details on the derivation of the model can be found in [23]. Thus, Fig. 2(a) and (b) represents, respectively, the unit cell of the structure under analysis and the circuit model that accounts for its electromagnetic response. In this circuit model:

1) The impedances $Z_{n}^{(0)}$ and $Z_{n}^{(1)}$ are the characteristic impedances of the transmission lines modeling the propagation of the $n$th order harmonic in medium (0) (free space) and (1) (dielectric substrate), with $|n| \leq$ $N$. The contribution of the $n$th harmonic inside the dielectric slab is modeled by the input impedance $Z_{n \text {,in }}^{(1)}$ 


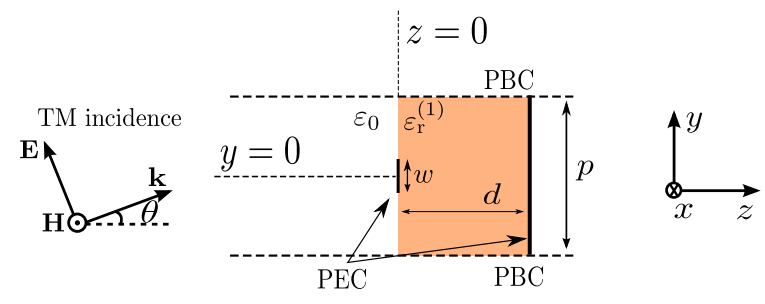

(a)

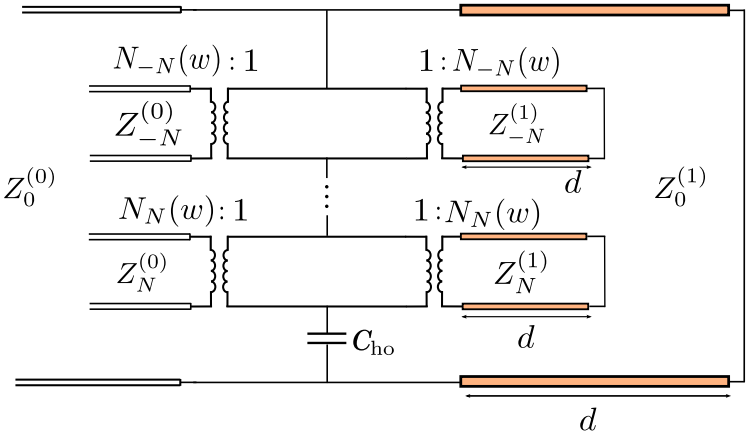

(b)

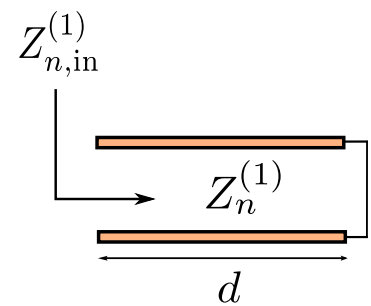

(c)

Fig. 2. (a) Lateral view of the unit cell. The upper and lower walls of the cell are periodic boundary conditions (PBCs). PEC stands for perfect electric conductor. (b) Equivalent circuit associated with the unit cell. (c) Input impedance seen from the discontinuity through a $n$ th-order short-terminated transmission line.

in Fig. 2(c). Their values are then given by

$$
\begin{aligned}
Z_{n}^{(i)} & =\frac{\eta_{0} \beta_{n}^{(i)}}{\varepsilon_{\mathrm{r}}^{(i)} k_{0}} \quad(i=0,1) \\
Z_{n, \text { in }}^{(1)} & =\mathrm{j} Z_{n}^{(1)} \tan \left(\beta_{n}^{(1)} d\right)
\end{aligned}
$$

with $\eta_{0}$ and $k_{0}$ being the intrinsic impedance and wavenumber in vacuum, $\varepsilon_{\mathrm{r}}^{(i)}$ the relative permittivity in medium $(i)$ [in our case, $\varepsilon_{\mathrm{r}}^{(0)}=1$ ], and $\beta_{n}^{(i)}$ the longitudinal $(z)$ component of the wavevector in medium $(i)$

$$
\beta_{n}^{(i)}=\sqrt{\varepsilon_{\mathrm{r}}^{(i)} k_{0}^{2}-\left(k_{\mathrm{t}}+k_{n}\right)^{2}} ; \quad k_{n}=\frac{2 \pi n}{p}
$$

where

$$
k_{\mathrm{t}}=k_{0} \sin \theta
$$

is the transverse component of the wavevector, with $\theta$ being the incidence angle.

2) The set of $N$ harmonics explicitly considered in the model comprises the few propagating diffraction orders as well as the lowest-order evanescent harmonic. This latter term is included to explicitly take into account the non trivial contribution to the frequency dependence of its associated impedance.

3) The transformers account for the coupling between the incident wave and high-order harmonics (analytical expressions for the turn ratios $N_{n}(w)$ can be found in [23]). Interestingly, the information about the strip width, $w$, only appears in these transformer ratios.

4) The capacitor $C_{\text {ho }}$ stands for the contribution of the remaining set of infinite high-order (ho) harmonics (i.e., harmonics with $|n|>N$ ). These harmonics are of TM nature and their cutoff frequencies are well above the operation frequency. An analytic expression for $C_{\mathrm{ho}}$ can be found in [23].

The validity range of the proposed equivalent circuit is determined by the ratios $w / p$ and $w / \lambda$ (with $\lambda$ being the wavelength in the dielectric medium). In our experience, the accuracy of the circuit model is guaranteed when those ratios are below 0.5 . Fortunately, these conditions are frequently found in many practical situations, even for operation frequencies beyond the onset of the grating lobes regime. This makes the proposed circuit model be a very efficient tool for the study of blazing, as it will be discussed in the following sections.

\section{Simplified CiRCUit FOR BRAGG Blazing}

Perfect Bragg blazing occurs when the reflected wave in medium (0) scatters back along the direction of incidence with equal power as the incident wave (no other diffraction orders appear). Complete power transfer from the incident wave to the $n=-1$ harmonic occurs when this harmonic diffracts exactly along the $n=0$ impinging direction. As already reported in [24], this phenomenon can accurately be accounted for by the circuit shown in Fig. 2(b). Even though the circuit topology may appear to be somewhat complex, a significant simplification can be obtained for Bragg blazing. The Bragg condition for constructive interference to be satisfied in this case is easily stated in terms of the transverse components of the wavevectors associated with the two involved harmonics $(n=0,-1)$

$$
k_{-1}+k_{\mathrm{t}}=-k_{\mathrm{t}} \Rightarrow k_{-1}=-2 k_{\mathrm{t}} .
$$

From (1)-(3), it is clear that (5) implies

$$
\beta_{0}^{(i)}=\beta_{-1}^{(i)} ; \quad Z_{0}^{(i)}=Z_{-1}^{(i)} ; Z_{0, \text { in }}^{(1)}=Z_{-1, \text { in }}^{(1)} .
$$

In addition, it can be shown that under this condition $\left|N_{-1}(w)\right|=1$ and therefore the transformers can be removed for the $n=-1$ harmonic. Since the $n=-1$ harmonic plays a special role in blazing, it will be hereafter considered separately from the rest of harmonics. The equivalent circuit of the strip-like discontinuity involved in our problem is then separated into a block for the $n=-1$ harmonic [boxed in dotted (red) line in Fig. 3(a)] plus another block that comprises the contribution of the next harmonic $(n=-2$ in Fig. 3(a); although the harmonic $n=1$ would play the same role as $n=-2$ at Bragg condition) as well as the capacitor accounting for the remaining high-order harmonics [boxed in dashed (blue) line]. The outer line in the dotted/red box, with realvalued characteristic impedance $Z_{0}^{(0)}$, acts as a simple loading 


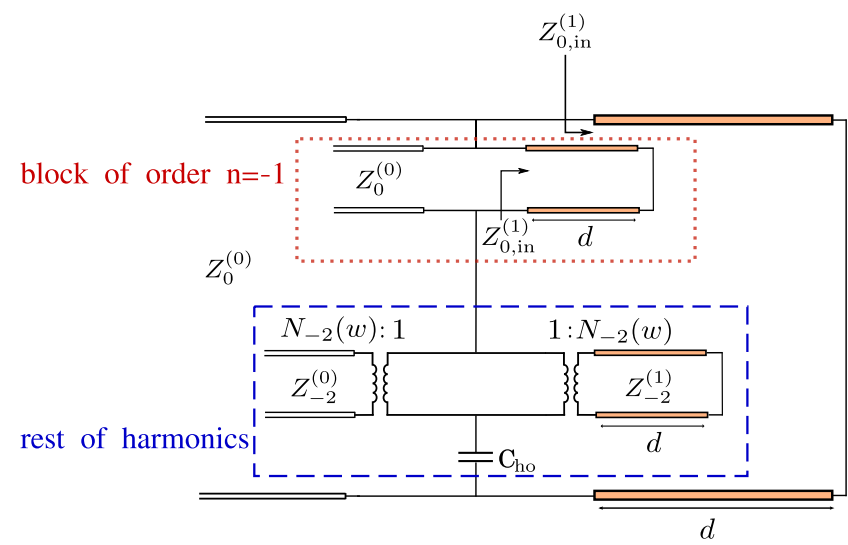

(a)

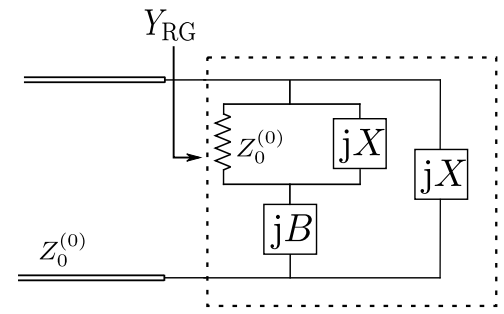

(b)

Fig. 3. (a) Equivalent circuit when the Bragg condition is fulfilled. (b) Simplified version of the circuit at blazing.

resistor. The inner line (grounded dielectric), on the contrary, yields a reactance for both the $n=0$ and $n=-1$ harmonics, given by

$$
\mathrm{j} X=Z_{0, \text { in }}^{(1)}=Z_{-1, \text { in }}^{(1)}=\mathrm{j} Z_{0}^{(1)} \tan \left(\beta_{0}^{(1)} d\right) .
$$

The above considerations allow us to simplify the circuit topology in Fig. 3(a) to that in Fig. 3(b). The capacitive or inductive nature of $X$ will depend on the sign of the tangent function in (7), which in turn comes determined by the dielectric slab thickness, $d$. For electrically thin slabs (the case we are interested in), the tangent function can be approximated by its argument, in such a way that

$$
\mathrm{j} X \approx \mathrm{j} \frac{\eta_{0}\left[\beta_{0}^{(1)}\right]^{2}}{\varepsilon_{\mathrm{r}}^{(1)} k_{0}} d
$$

namely, a purely inductive impedance.

The circuit in Fig. 3(b) involves an additional impedance, $\mathrm{j} B$, which accounts for the blue-boxed contributions of the remaining harmonics and that can be written as

$$
\mathrm{j} B=\frac{\left[N_{-2}(w)\right]^{2}}{Y_{-2}^{(0)}+Y_{-2, \text { in }}^{(1)}}+\frac{1}{\mathrm{j} \omega C_{\mathrm{ho}}} .
$$

The capacitive or inductive nature of this impedance only depends on the behavior of the shunt connection associated with the $n=-2$ harmonic (remind that the contribution of higher order harmonics is always capacitive and is already packed into $C_{\text {ho }}$ ). Since the $n=-2$ harmonic is evanescent in the air region (otherwise more than one diffraction lobe would be present), the blazed frequency should be set below the onset frequency of the $n=-2$ harmonic in air. Thus, the characteristic impedance $Z_{-2}^{(0)}$ behaves as the following capacitive reactance:

$$
Z_{-2}^{(0)}=-\mathrm{j} \frac{\eta_{0}\left|\beta_{-2}^{(0)}\right|}{k_{0}}
$$

This assumption does not apply to the same harmonic inside the dielectric slab, where the corresponding fields can be either evanescent or propagative. In any case, the input impedance is given by

$$
Z_{-2, \text { in }}^{(1)}= \begin{cases}-\mathrm{j} \frac{\eta_{0}\left|\beta_{-2}^{(1)}\right|}{\varepsilon_{\mathrm{r}} k_{0}} \tanh \left(\left|\beta_{-2}^{(1)}\right| d\right) & \text { (a): below cutoff } \\ \mathrm{j} \frac{\eta_{0} \beta_{-2}^{(1)}}{\varepsilon_{\mathrm{r}} k_{0}} \tan \left(\beta_{-2}^{(1)} d\right) & \text { (b): above cutoff }\end{cases}
$$

in such a way that its capacitive/inductive nature depends on whether the harmonic is either evanescent or propagative at the operation frequency. In the first case [see (11a)], $Z_{-2 \text {,in }}^{(1)}$ is a capacitive load, so that the overall contribution of $n=$ -2 will always be capacitive. This contribution is series connected to $C_{\mathrm{ho}}$, thus giving place to a global capacitive impedance [jB in Fig. 3(b)]. However, if $Z_{-2 \text {,in }}^{(1)}$ is given by (11b), the global behavior of $\mathrm{j} B$ might become inductive [note that the inductive nature of this impedance can turn into capacitive when the tangent function changes sign in (11b)]. This strong dependence caused by the tangent function is a problem when trying to establish simple design recipes for the blazing grating. However, if an electrically thin dielectric slab is assumed $(d \ll \lambda), Z_{-2 \text {,in }}^{(1)}$ will be a very small impedance, thus making that the impedance of the blue/dashed-boxed block can be well approximated by

$$
\mathrm{j} B \approx \frac{1}{\mathrm{j} \omega C_{\mathrm{ho}}} .
$$

For thick dielectric slabs, the circuit model as well as its frequency behavior becomes more complicated. Fortunately, we are interested in gratings as electrically thin as possible, in such a way that the described approximation is fairly accurate.

In the simplified equivalent circuit sketched in Fig. 3(b), the equivalent admittance seen from the discontinuity toward the right-hand side $\left(Y_{\mathrm{RG}}\right)$ can be written as

$$
Y_{\mathrm{RG}}=\frac{Y_{0}^{(0)}\left(1+\frac{B}{X}\right)-\mathrm{j} \frac{1}{X}\left(2+\frac{B}{X}\right)}{1+\frac{B}{X}+\mathrm{j} B Y_{0}^{(0)}} .
$$

From a circuit point of view, perfect Bragg blazing essentially means perfect matching in such equivalent circuit, that is

$$
Y_{0}^{(0)}=Y_{\mathrm{RG}} .
$$

This condition requires the imaginary part of $Y_{\mathrm{RG}}$ to vanish and its real part to be identical to the characteristic admittance of the input line. Simple inspection of (13) leads to the following straightforward particular solution of (14):

$$
B=-X \text { and } X=Z_{0}^{(0)} .
$$

The above equalities indicate that perfect Bragg blazing occurs at resonance $(B=-X)$ provided that $Z_{0}^{(0)}=\left|Z_{0, \text { in }}^{(1)}\right|$. 
Resonance itself does not ensure perfect blazing since some specular reflection of the $n=0$ harmonic would be observed if the real part of $Y_{\mathrm{RG}}$ at resonance does not match $Y_{0}^{(0)}$. It should be noticed here that the condition to cancel the imaginary part of the equivalent impedance is not unique. The possibility of having more solutions will be discussed in Section V.

\section{Design Procedure For BRAgG Blazing}

In this section, a simple recipe will be outlined to design a strip-like grating exhibiting high Bragg blazing efficiency. The aim of the proposed procedure is to find the optimum values of the geometrical parameters of the structure that satisfy the perfect blazing conditions given in (15). The proposed steps are the following.

1) Once the operation frequency $\left(f_{b}\right)$ and incidence angle $(\theta)$ for blazing are specified, the period is determined from the Bragg condition (5) as

$$
k_{-1}=-2 k_{\mathrm{t}} \Rightarrow p=\frac{c}{2 f_{\mathrm{b}} \sin \theta} .
$$

It is important to ensure that no propagating harmonics other than $n=0,-1$ exist in the air region for the chosen values of $f_{\mathrm{b}}, \theta$, and $p$. The condition for the $n$th harmonic being evanescent is

$$
\left|k_{n}+k_{t}\right|>k_{0}
$$

Using the Bragg condition (5) in (17) leads to

$$
\sin \theta>\frac{1}{|2 n+1|}
$$

Therefore, in order to achieve perfect Bragg blazing, it is clear that the incidence angle must satisfy

$$
\theta>\arcsin (1 / 3)=19.47^{\circ} \text {. }
$$

2) Taking into account that $Z_{0}^{(0)}=\eta_{0} \cos \theta$ and combining (2), (7), and (15), it is obtained

$$
k_{0} \cos \theta=\frac{\beta_{0}^{(1)} \tan \left(\beta_{0}^{(1)} d\right)}{\varepsilon_{\mathrm{r}}^{(1)}} .
$$

The above condition can be fulfilled by many different combinations of $\varepsilon_{\mathrm{r}}^{(1)}$ and $d$ ( $d$ being electrically small). For a given value of $\varepsilon_{\mathrm{r}}^{(1)}$, the appropriate dielectric thickness can easily be determined from (20), and vice versa.

3) Finally, for a given combination of $\varepsilon_{\mathrm{r}}^{(1)}$ and $d$, a suitable value of $w$ should be calculated in order to satisfy $B=-X$, which according to (7) and (9) leads to

$$
\frac{\left[N_{-2}(w)\right]^{2}}{Y_{-2}^{(0)}+Y_{-2, \text { in }}^{(1)}}+\frac{1}{\mathrm{j} \omega C_{\mathrm{ho}}(w)}=-Z_{0}^{(1)} \tan \left(\beta_{0}^{(1)} d\right) \text {. }
$$

This is a transcendental equation that can be numerically solved using any appropriate root-finding method.

Next, the above steps will be applied to design a prototype of grating. The goal is to achieve perfect blazing at $f_{\mathrm{b}}=$ $8 \mathrm{GHz}$ with $\theta=35^{\circ}$ and $\varepsilon_{\mathrm{r}}^{(1)}=6.15$. From (16), the required period is $p=32.7 \mathrm{~mm}$ and, from (20), the dielectric thickness should be $d=2.78 \mathrm{~mm}$. Finally, using (21), the required value of the strip width is $w=5.10 \mathrm{~mm}$. The computed scattering response of the prototype is plotted in Fig. 4(a), where the normalized values of the SR power and the power carried by the $n=-1$ harmonic are represented. At the target frequency, the reflected power is lower than $-40 \mathrm{~dB}$, so that practically all the power has been transferred to the $n=-1$ harmonic. Excellent agreement between analytical and numerical MoM results can be appreciated. As an additional check of condition (15), Fig. 4(b) shows the real and imaginary parts of the complex impedance loading the transmission line associated with the impinging $n=0$ harmonic (i.e., $Z_{\mathrm{RG}}=Y_{\mathrm{RG}}^{-1}$ ). Note that, as expected at the blazing frequency, the real part matches the value of the characteristic impedance of the input line and the imaginary part vanishes. This condition ensures perfect transfer of the impinging $n=0$ order to the diffracted $n=-1$ order. Fig. 4(c) shows the frequency behavior of the individual impedances associated with the $n=0,-1$ harmonics around $8 \mathrm{GHz}$. All these values intersect at $8 \mathrm{GHz}$, as required by the model to achieve perfect Bragg blazing operation [see (6) and (15)]. The value of the impedances at the intersection point is approximately $310 \Omega$. This value is reached by the impedance $B$ at $8 \mathrm{GHz}$, as shown in Fig. 4(d). As required, $B$ is a negative reactance.

\section{General Resonance Mechanism FOR BRAGG BLAZING}

In (15), the condition for Bragg blazing was posed as $B=-X$ at the blazing frequency. Nevertheless, this condition is sufficient to ensure resonance but not necessary. Actually, since the matching condition for perfect Bragg blazing is given by (14), the following more general version of the resonance requirement can be obtained combining (13) and (14):

$$
B=\frac{(2 / X)}{\left[Y_{0}^{(0)}\right]^{2}+(1 / X)^{2}} \text {. }
$$

The value of $Y_{0}^{(0)}$ is defined once the incidence angle is imposed. Note that the resonance condition (22) can be satisfied by a variety of pairs of $B$ and $X$ values. These combinations correspond to specific choices of parameters $w$ and $d$. According to the definition of $X$ and $B$ in (7) and (9), condition (22) can be rewritten as

$$
\begin{aligned}
\operatorname{Im}\left(\frac{\left[N_{-2}\right]^{2}}{Y_{-2}^{(0)}+Y_{-2, \text { in }}^{(1)}}\right. & \left.+\frac{1}{\mathrm{j} \omega C_{\mathrm{ho}}}\right) \\
& =-2 \frac{Y_{0}^{(1)} \cot \left(\beta_{0}^{(1)} d\right)}{\left[Y_{0}^{(0)}\right]^{2}+\left[Y_{0}^{(1)} \cot \left(\beta_{0}^{(1)} d\right)\right]^{2}} .
\end{aligned}
$$

A design procedure incorporating (23) would be identical to the one reported in Section IV, but substituting the transcendental equation (21) by (23) in the third step. It is worth noting that (22) indicates that, for a set of values of $f_{\mathrm{b}}, \theta$, and $\varepsilon_{\mathrm{r}}^{(1)}$, perfect blazing along the incidence direction can be achieved for many different combinations of $w$ and $d$. Examples of the frequency response for several of those combinations are depicted in Fig. 5(a). In this figure, the power carried by the $n=-1$ harmonic is plotted as a function of frequency for six 


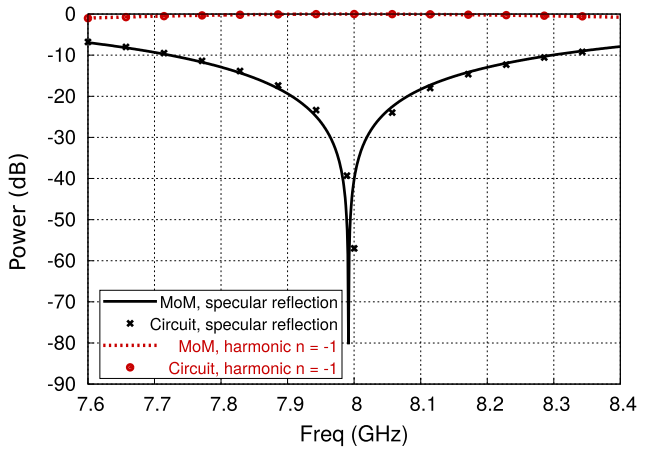

(a)

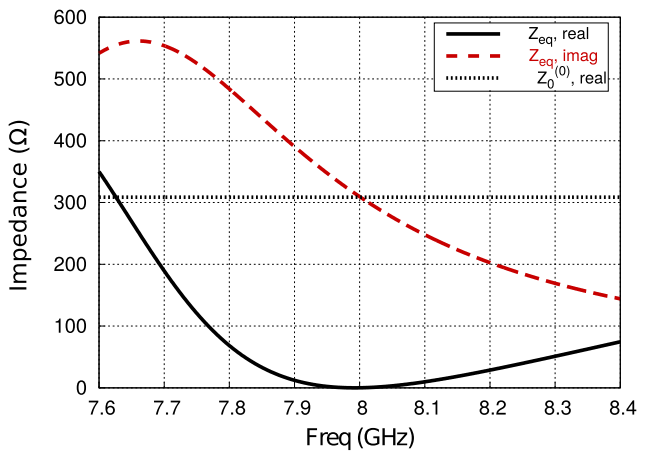

(b)

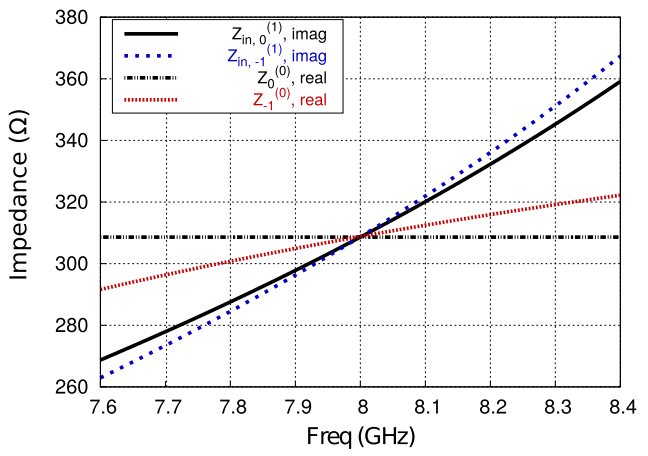

(c)

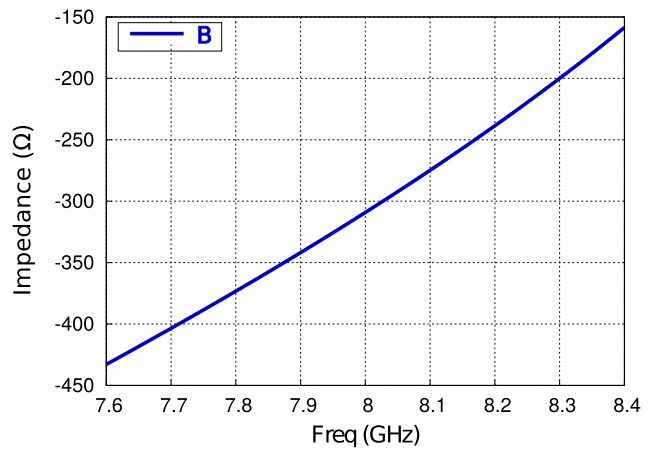

(d)

Fig. 4. (a) Power associated with specular reflection (black solid line) and transferred to the harmonic of order $n=-1$ (red dotted line) computed with method of moments $(\mathrm{MoM})$ and circuit model (black crosses and red dots). (b) Real (black solid line) and imaginary (red dashed line) parts of the impedance loading the transmission line representing the $n=0$ harmonic. (c) Frequency dependence of the impedances associated with the harmonics $n=0,-1$ in the media (0) and (1). (d) Frequency dependence of the impedance $B$. Structure parameters: $p=32.7 \mathrm{~mm}, w=5.10 \mathrm{~mm}$, $d=2.78 \mathrm{~mm}, \varepsilon_{\mathrm{r}}^{(1)}=6.15$, and $\theta=35^{\circ}$.

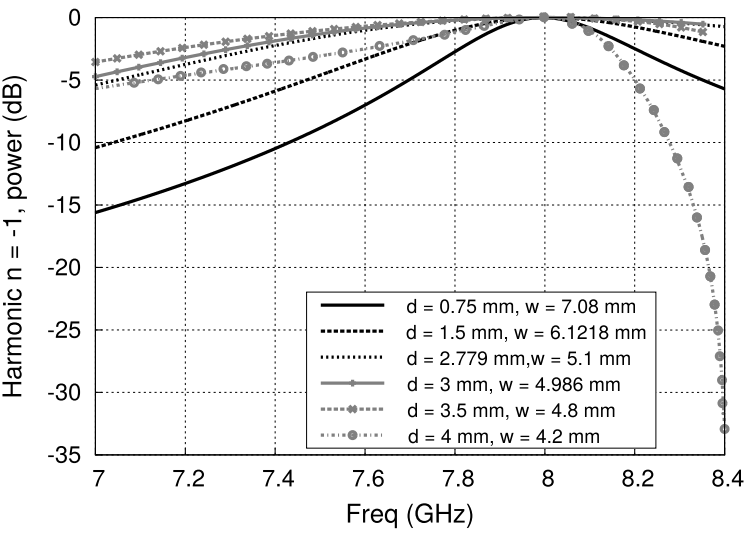

(a)

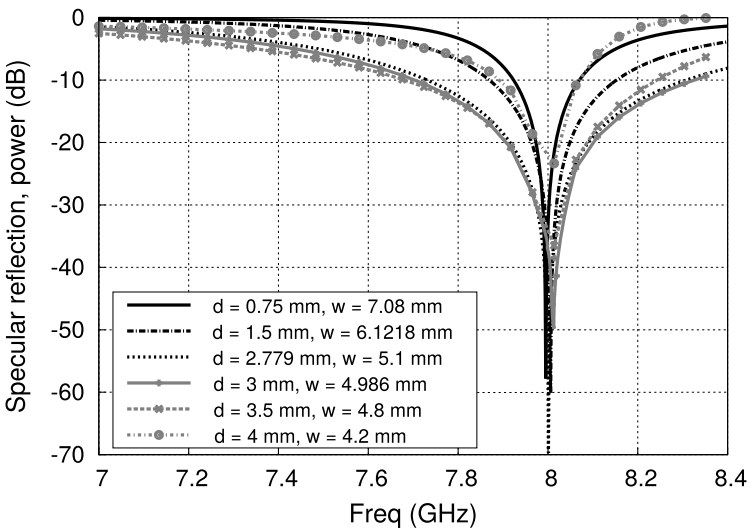

(b)

Fig. 5. (a) Power transferred to the harmonic of order $n=-1$. (b) Specularreflection power for several designs sharing the value of $f_{b}(8 \mathrm{GHz})$ and the following dimensional and electrical parameters: $p=32.7 \mathrm{~mm}, \theta=35^{\circ}$, $\varepsilon_{\mathrm{r}}^{(1)}=6.15$. Note that for each value of $d$, an appropriate value of $w$ has been chosen.

different geometrical designs. The design specifications are the ones considered in Fig. 4, but several different pairs of $w$ and $d$ satisfying (23) have been compared. As required, the maximum blazing power is always attained at $8 \mathrm{GHz}$. Note that it means that, for a given commercial substrate with specific values of $d$ and $\varepsilon_{\mathrm{r}}^{(1)}$, it is always possible to find a value of $w$ leading to perfect blazing. However, it should be noted that the bandwidth associated with each configuration is different. Similar conclusions can be inferred from Fig. 5(b), where the specular-reflection power has been plotted for the same frequency range.

Provided an incidence angle and frequency are chosen for perfect blazing, an interesting question arises on the optimum choice of the pair $(w, d)$ that leads to the broadest bandwidth. In Fig. 6(a), the evolution of the fractional bandwidth for a given value of permittivity and several different dielectric thicknesses is shown (the fractional bandwidth is defined as the percentage ratio of the frequency band for which the specularreflection level is less than $-10 \mathrm{~dB}$ and the corresponding central frequency). Of course, for each dielectric thickness, there is a particular value of $w$ that gives rise to perfect Bragg blazing [this value is plotted in Fig. 6(b)]. Note that 


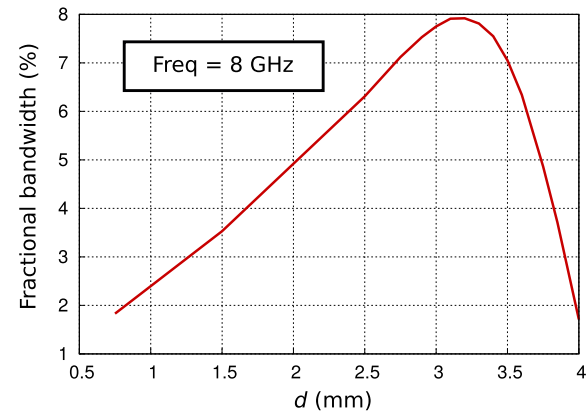

(a)

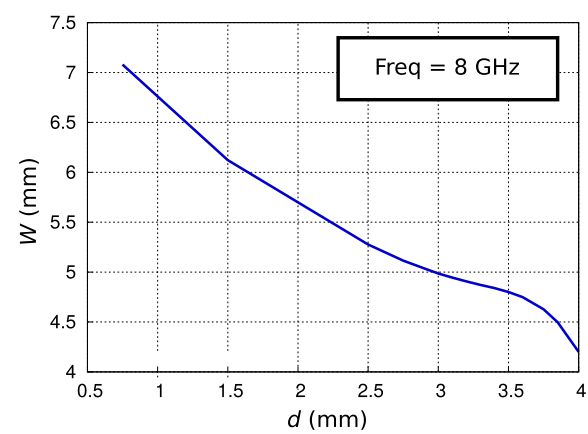

(b)

Fig. 6. (a) Fractional bandwidth for the specular-reflection power (under $-10 \mathrm{~dB}$ ). The central frequency is $8 \mathrm{GHz}$. The figure shows the evolution of the bandwidth in terms of the dielectric thickness. (b) Corresponding strip width value $(w)$ for each of the dielectric thicknesses used in (a). Common parameters: $p=32.7 \mathrm{~mm}, \theta=35^{\circ}, \varepsilon_{\mathrm{r}}^{(1)}=6.15$.

the bandwidth is small for both very thin and relatively thick substrates, reaching an optimum value (maximum bandwidth) at about $d=3.3 \mathrm{~mm}$. This means that, for a given substrate dielectric constant, we can select the dielectric thickness closest to the optimum one if a reasonably wide bandwidth is required. The proposed circuit model also helps to make such choice.

\section{MODEL FOR TE INCIDENCE}

In Sections II-V, only TM polarization of the impinging wave has been considered and now it will explore the possibility of achieving perfect blazing for TE polarization. Actually, circuit models similar to those reported for TM polarization are also available for the TE case [23]. According to the study presented in [23], the topology of the circuit models for TE polarization is identical to the one shown in Fig. 2 (or Fig. 3). However, since only TE harmonics are scattered by the strip grating, TM modal impedances should be replaced by TE ones (i.e., $Z_{n}^{(i)}=\eta_{0} k_{0} / \beta_{n}^{(i)}$ ) in the whole formulation. The values of $N_{n}(w)$ should also be changed due to the drastically different current profile induced on the metal strips when TE excitation is considered [23]. The most relevant difference with the TM case, in the context of this discussion, is that the capacitance $C_{\mathrm{ho}}$ accounting for high-order harmonic contributions has to be replaced with an inductance $L_{\text {ho }}$ whose explicit value can be found in [23]. It means that the nature of the reactive impedance, $\mathrm{j} B$, in the simplified circuit shown in Fig. 3(b) is inductive $(B>0)$ for the TE case, in such a way

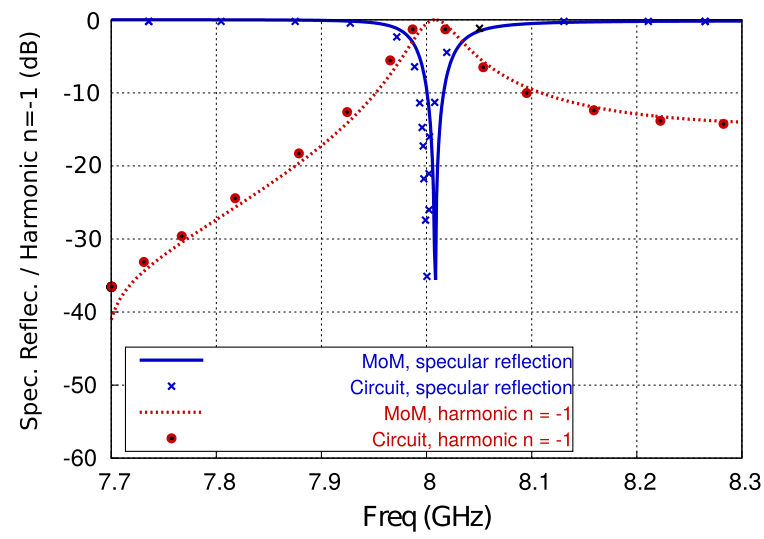

Fig. 7. SR power (blue solid line) and power transmitted to the $n=-1$ harmonic (red dotted line) for TE incidence (blue crosses and red dots have been obtained with the analytical equivalent circuit). Parameters: $\theta=35^{\circ}$, $\varepsilon_{r}=6.15, w=5.1 \mathrm{~mm}$, and $d=8.85 \mathrm{~mm}$.

that resonance is not possible for $X>0$. In other words, for TE polarization, there is no possibility of perfectly matching the $n=-1$ harmonic (blazing) if the thickness of the substrate is small, since in such case $X$ is always positive. However, if this restriction is relaxed and the substrate is allowed to be electrically thick, negative values of $X$ can be obtained for $\pi / 2<\beta_{0}^{(1)} d<3 \pi / 2$ [see (7)]. The resonance condition is, once again, expressed by (22). Let us consider a simple example to illustrate this possibility. For an incidence angle of $\theta=35^{\circ}$ and blazed frequency $f_{b}=8 \mathrm{GHz}$, the period of the structure should be $p=32.7 \mathrm{~mm}$, according to the previously explained procedure. The next step is to fulfill the resonance condition given by (22). For a given permittivity we can find, as explained previously for TM polarization, a variety of pairs $(w, d)$ satisfying $(22)$. For fixed values of $w$ and $\varepsilon_{r}$ we can numerically solve (22) for $d$. Let us take the particular values $\varepsilon_{r}=6.15$ and $w=5.1 \mathrm{~mm}$. The smallest value of $d$ fulfilling condition (22) turns to be $d=8.85 \mathrm{~mm}$. For this set of values, in Fig. 7 we have plotted the SR power along with the power transmitted to the $n=-1$ harmonic. Note that the circuit model and MoM results show a reasonably good agreement over the whole explored frequency band. Thus, in principle, perfect blazing is possible for TE polarization. However, due to the requirement of thick substrates, the bandwidth of perfect blazing is much smaller than the one achievable for TM incidence. This could be a serious drawback for practical applications of this particular structure when used for TE polarization. The circuit model provides a clear explanation of the reasons for reducing the bandwidth and can guide the designer to find new structures overcoming this drawback.

\section{Perfect OfF-Bragg Blazing}

Up to this point, only perfect back reflection along the incidence direction (Littrow case) has been considered. However, one might be interested in achieving perfect blazing along a particular direction $\theta^{\prime}$ different from the incidence direction $\theta$ (off-Bragg blazing). Note that the blazed angle $\theta^{\prime}$ is actually determined by the ratio between the transverse and longitudinal components of the wavevector associated with the 


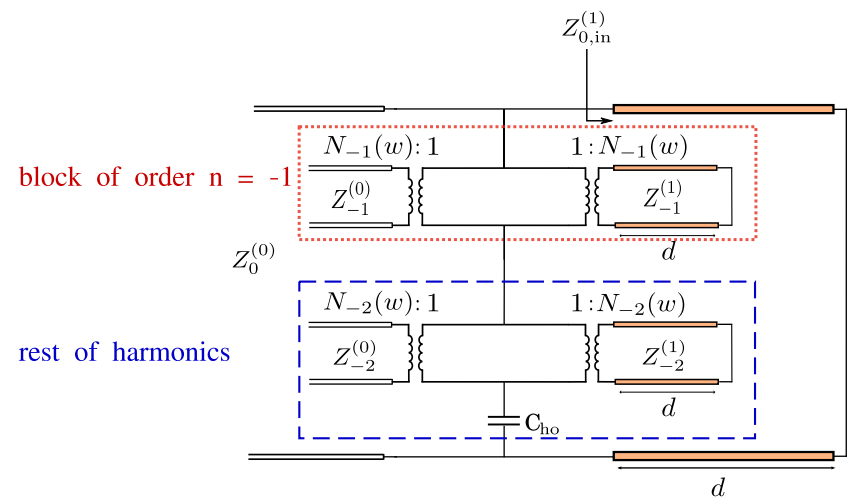

(a)

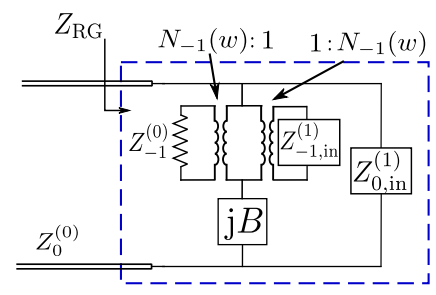

(b)

Fig. 8. (a) Circuit model to account for off-Bragg blazing. (b) Equivalent circuit considering $\mathrm{j} B$.

$n=-1$ diffracted harmonic; namely

$$
\tan \theta^{\prime}=\frac{k_{0} \sin \theta-2 \pi / p}{\beta_{-1}^{(0)}} .
$$

By using the formal expressions of these parameters and after some manipulations, (24) can be rewritten as follows:

$$
\tan \theta^{\prime}=\frac{f p \sin \theta-c}{\sqrt{[f p \cos \theta]^{2}-c^{2}+2 c p f \sin \theta}} .
$$

This equation shows how the blazed angle depends on the incidence angle, the operation frequency, and the period of the grating. It would allow us, for instance, to fix the values of $\theta$, $\theta^{\prime}$, and $f_{\mathrm{b}}$ and use (25) to determine the proper value of the period $p$. It is worth mentioning that there is no information about the geometry of the structure in (25) (except the period). The period together with the incidence angle controls the onset of a certain diffraction order, while the propagation direction of such diffraction order is controlled by the operation frequency. However, no conditions about the coupling level between the incident harmonic and the $n=-1$ harmonic can be enforced by (25). The power transfer from the incident harmonic to the $n=-1$ harmonic is dictated by the geometry of the cell (dielectric thickness, $d$, and strip width, $w$ ) and the relative permittivity of the substrate, $\varepsilon_{\mathrm{r}}^{(1)}$. These parameters can be adjusted to suppress the specular reflection of the impinging harmonic and transfer all the power to the $n=-1$ harmonic, which will propagate along the $\theta^{\prime}$ direction. This perfect blazing condition is discussed next.

\section{A. Condition to Achieve Perfect Off-Bragg Blazing}

Once the period of the structure has been fixed, and the blazed frequency and incidence/blazed angles have been chosen, the next step in the design procedure consists of tuning $w, d, \varepsilon_{\mathrm{r}}^{(1)}$ to reach a perfect power transfer between the harmonics under consideration. Fortunately, from the equivalent circuit point of view, this is again just an impedance matching problem involving the input line, $Z_{0}^{(0)}$, and the impedance loading such transmission line, $Z_{\mathrm{RG}}$; that is, (14). The corresponding equivalent circuit is represented in Fig. 8(a) and its simplified version in Fig. 8(b). According to this simplified circuit, the matching condition can now be written as

$$
Y_{0}^{(0)}\left[=Y_{\mathrm{RG}}=1 / Z_{\mathrm{RG}}\right]=\frac{1}{\mathrm{j} B+\frac{\left[N_{-1}(w)\right]^{2}}{Y_{-1}^{(0)}+Y_{-1, \text { in }}^{(1)}}}+Y_{0, \text { in }}^{(1)}
$$

with

$$
\mathrm{j} B=-\mathrm{j} \frac{1}{\omega C_{\mathrm{ho}}}+\frac{\left[N_{-2}(w)\right]^{2}}{Y_{-2}^{(0)}+Y_{-2, \text { in }}^{(1)}} .
$$

Note that, once again, the dynamical frequency contribution of the lowest-order evanescent harmonic $(n=-2)$ has been explicitly taken into account since this harmonic might operate close to its onset frequency. The contribution of the remaining evanescent harmonics is included in the frequency-independent high-order capacitance $C_{\mathrm{ho}}$.

Once the parameters involved in (25) have been fixed, the variables to be considered in (26) are just $w, d$, and $\varepsilon_{\mathrm{r}}^{(1)}$. For instance, fixing the permittivity value of the dielectric slab, an appropriate pair of values $w, d$ satisfying (26) can readily be found by means of a numerical root-searching algorithm. A physical interpretation of the solution to (26) can be inferred by noting that, since the left-hand member of (26) is real valued, the right-hand side has to be real too; namely, the imaginary part of the right-hand side of (26) has to vanish at the matching frequency. It means that the structure has to operate at resonance, as it was also found for Bragg blazing. Perfect blazing is, again, promoted by a resonance phenomenon. It is worth mentioning here that, even though only TM cases are treated in this section, TE polarization can be handled in a similar manner. However, in this latter case, thick substrates are required and narrow band operation is achieved, as discussed in Section VI.

\section{B. Numerical Examples}

In order to validate the above remarks, two different designs are carried out according to the following specifications:

Case 1: $\theta=45^{\circ}, \theta^{\prime}=30^{\circ}, p=30 \mathrm{~mm}$, and $\varepsilon_{\mathrm{r}}^{(1)}=10.2$.

With these specifications, the blazed frequency $\left(f_{\mathrm{b}}\right)$ can be computed by using (25) and the dielectric thickness and strip width by using (26). The resulting values are $f_{\mathrm{b}}=$ $8.28 \mathrm{GHz}, w=3.76 \mathrm{~mm}$, and $d=1.96 \mathrm{~mm}$. The resulting specular-reflection power and the power carried by the $n=-1$ harmonic are both plotted in Fig. 9(a). A specularreflection minimum $(\sim-80 \mathrm{~dB})$ appears at the calculated blazed frequency, which corresponds to a maximum of power associated with the $n=-1$ harmonic. The frequency behavior of the impedance $Z_{\mathrm{RG}}$ is plotted in Fig. 9(b). As expected, this figure shows the zero-crossing of the imaginary part of $Z_{\mathrm{RG}}$ at the same frequency at which the real part of $Z_{\mathrm{RG}}$ coincides with the characteristic impedance of the input line. 


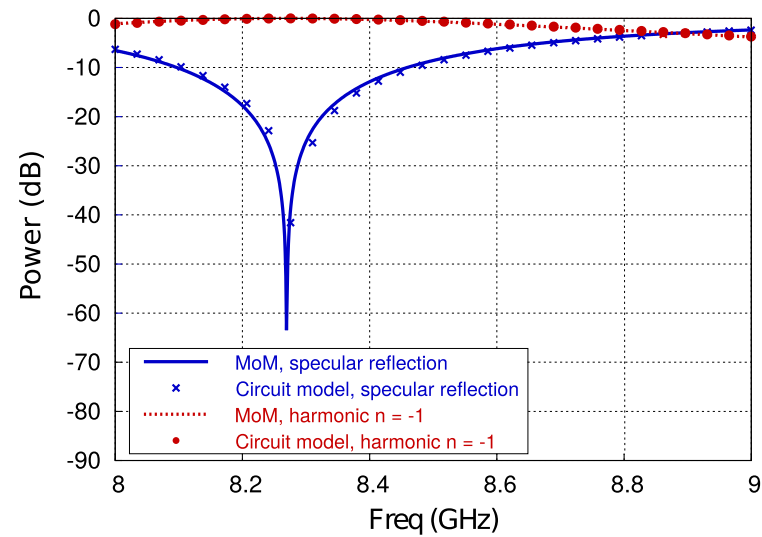

(a)

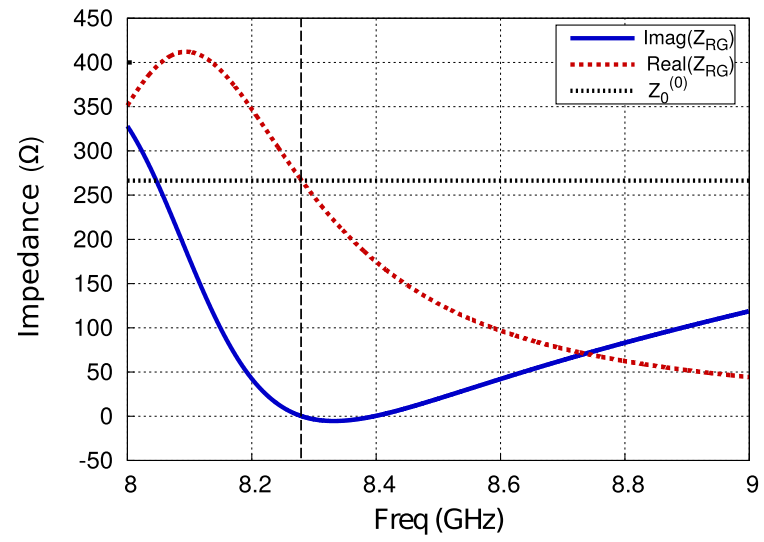

(b)

Fig. 9. (a) Power carried by the SR wave (blue solid line) and the harmonic of order $n=-1$ (red dotted line). Blue crosses and red dots have been computed using the analytical circuit model. Structure parameters: $p=30 \mathrm{~mm}$, $w=3.761 \mathrm{~mm}, d=1.96 \mathrm{~mm}, \varepsilon_{\mathrm{r}}^{(1)}=10.2, \theta=45^{\circ}, \theta^{\prime}=30^{\circ}$. (b) Frequency behavior of the real (dotted red line) and imaginary (solid blue line) parts of $Z_{\mathrm{RG}}$. The horizontal line represents the characteristic impedance of the input line and the vertical black dashed line indicates the blazed frequency.

This frequency is precisely the blazed frequency, as it can be seen in Fig. 9(a). Likewise the previous cases, the results provided by the equivalent circuit are compared with those obtained by an in-house MoM showing an excellent agreement.

Case 2: $\theta=20^{\circ}, \theta^{\prime}=40^{\circ}, \varepsilon_{\mathrm{r}}^{(1)}=7$, and $f_{\mathrm{b}}=7.5 \mathrm{GHz}$.

The geometrical parameters that satisfy these specifications can be determined using the same procedure as before. Since the blazed frequency has been initially fixed in this case, the combined use of (25) and (26) gives the following values: $p=40.59 \mathrm{~mm}, w=5.21 \mathrm{~mm}$, and $d=2.21 \mathrm{~mm}$. The corresponding response is plotted in Fig. 10(a), where it is found that the specular reflection reaches a deep minimum at the desired frequency. In Fig. 10(b), the frequency behavior of the real and imaginary parts of $Z_{\mathrm{RG}}$ is also shown, with the expected behavior of the real and imaginary parts of $Z_{\mathrm{RG}}$.

\section{Off-Bragg Blazing Points}

The resonance mechanism to achieve perfect blazing has been linked to the following two conditions: 1) the imaginary part of $Y_{\mathrm{RG}}$ in (26) must vanish (resonance) and 2) the real

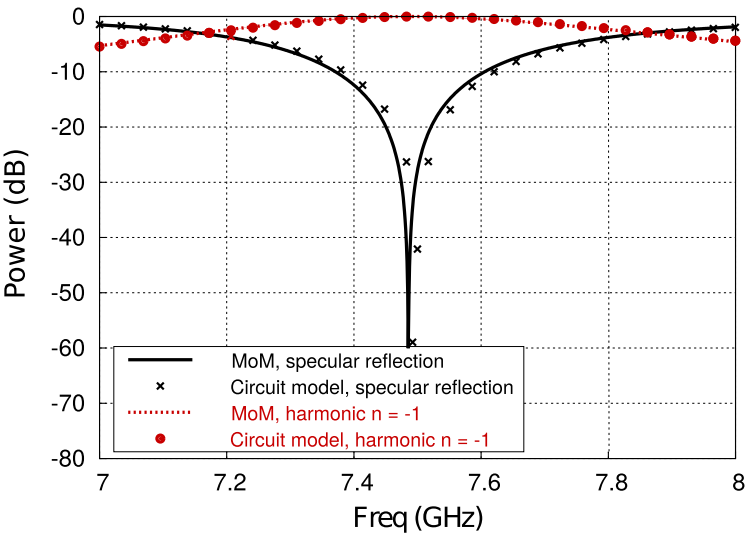

(a)

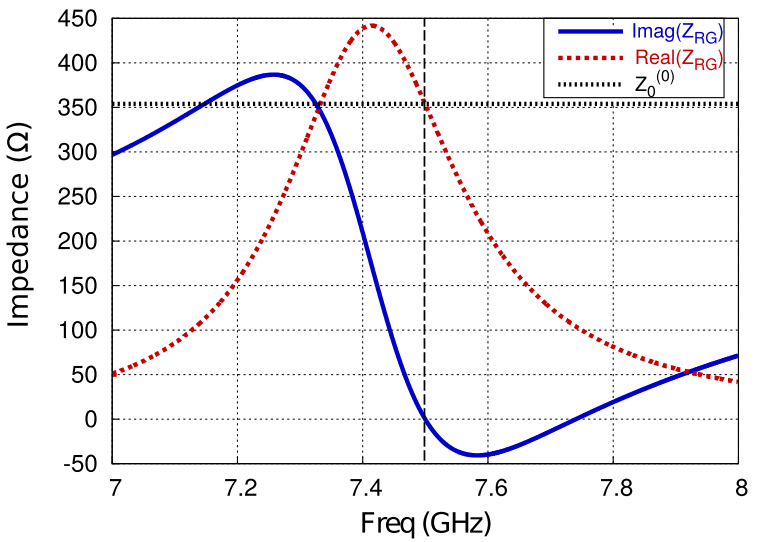

(b)

Fig. 10. (a) Power carried by the SR wave (black solid line) and the harmonic of order $n=-1$ (red dotted line). Crosses and dots correspond to the equivalent circuit model. Structure parameters: $p=40.589 \mathrm{~mm}$, $w=5.21 \mathrm{~mm}, d=2.2102 \mathrm{~mm}, \varepsilon_{\mathrm{r}}^{(1)}=7.0, \theta=20^{\circ}, \theta^{\prime}=40^{\circ}$. (b) Frequency behavior of the real (red dotted line) and imaginary (blue solid line) parts of $Z_{\mathrm{RG}}$. The horizontal dotted line represents the characteristic impedance of the input line, and the vertical dashed line indicates the blazed frequency.

part of $Y_{\mathrm{RG}}$ must be equal to $1 / Z_{0}^{(0)}$. The application of such conditions provides a systematic design procedure that can be very conveniently carried out with the already proposed equivalent circuit. In order to further corroborate this fact, the numerical results reported in [19, Fig. 4(a)] are now reproduced in Fig. 11 using the analytical circuit model. In this figure, the behavior of the specular-reflection coefficient is shown in terms of the operation frequency and incidence angle for the same grating configurations but with three different strip width, $w=2.7,3.4$, and $4.1 \mathrm{~mm}$. It can be checked that the agreement of the ECA results with the numerical results in $[19$, Fig. 4(a)] is excellent, with the low-reflection regions being well captured by the circuit model. In Fig.11, minima of specular reflection are observed at the points where the Bragg condition is fulfilled. Clearly, the blazed frequency (or blazed frequency band) shifts as $w$ varies following the Bragg line in the frequency-angle plane. [The Bragg line was defined in [19] and here corresponds to (16); namely, $f_{\mathrm{b}}=c /(2 p \sin \theta)$.] However, as stated in [19], two unexpected high-blazing peaks appear out of the Bragg line in the plot corresponding to $w=$ $4.1 \mathrm{~mm}$; these off-Bragg blazing points are also well captured 

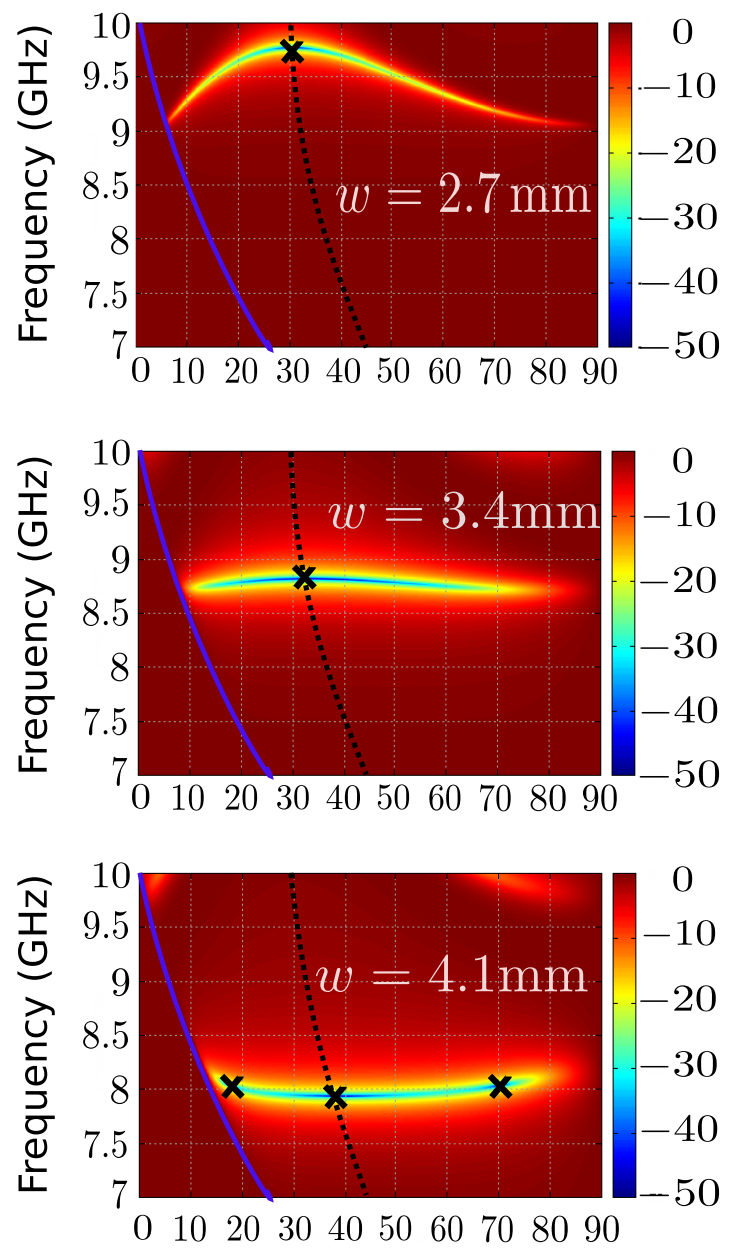

Incidence angle $\theta$ (Degrees)

Fig. 11. Equivalent-circuit results for the reflection coefficient (expressed in $\mathrm{dB}$ as a map of color) as a function of frequency and incidence angle for the same configurations considered in [19, Fig. 4(a)]. Blue solid curve marks the boundary between the nondiffractive and the diffractive regimes. The black dotted curve corresponds to the Bragg line.

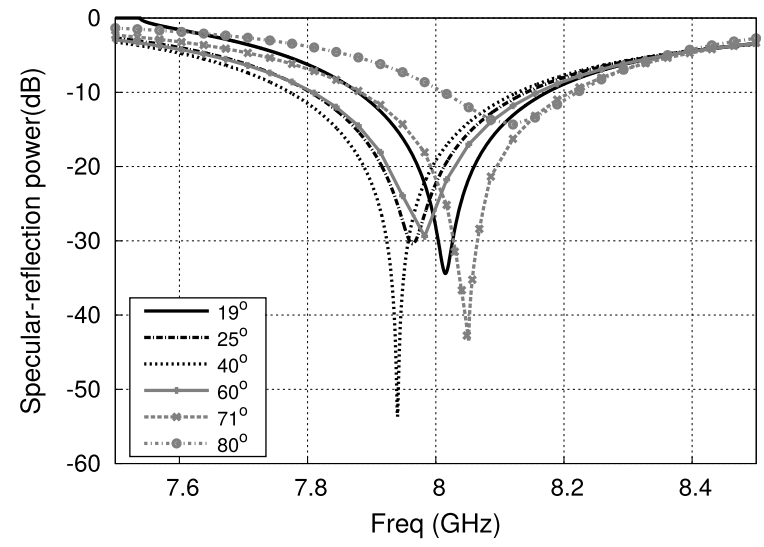

Fig. 12. Specular-reflection power for six different incidence angles for the structure considered in Fig. 11(c).

by the circuit model. A further study of this fact is shown in Fig. 12 where the reflection coefficient is plotted for several

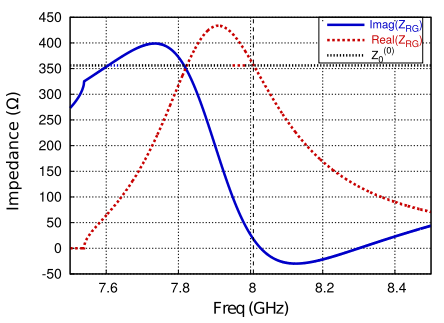

(a)

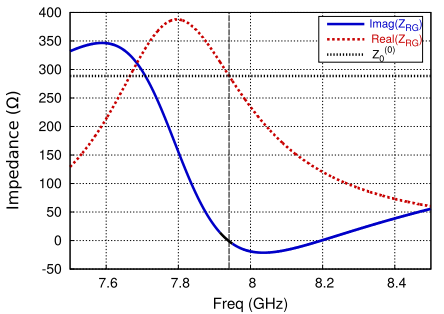

(c)

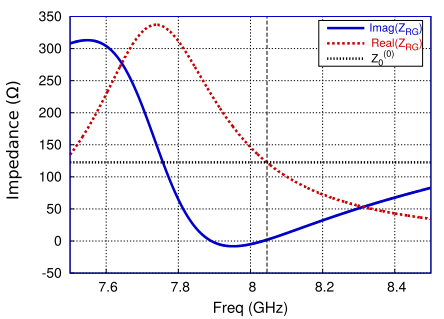

(e)

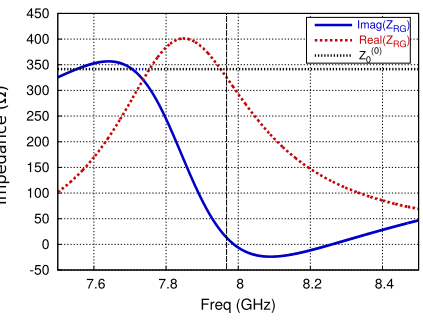

(b)

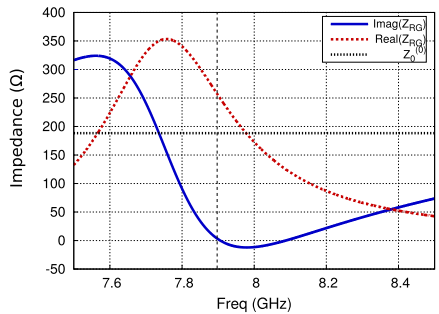

(d)

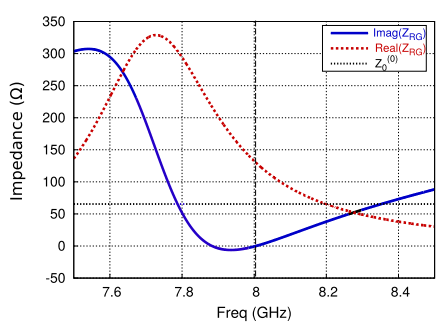

(f)
Fig. 13. Frequency behavior of the real (dotted red line) and imaginary (solid blue line) parts of $Z_{\mathrm{RG}}$ for the structure considered in Fig. 12. The dotted black horizontal line represents the value of $Z_{0}^{(0)}$ and the dashed black vertical line indicates the resonance frequency. (a) $\theta=19^{\circ}$. (b) $\theta=25^{\circ}$. (c) $\theta=40^{\circ}$. (d) $\theta=60^{\circ}$. (e) $\theta=71^{\circ}$. (f) $\theta=80^{\circ}$.

incidence angles around $f=8 \mathrm{GHz}$ for the structure considered in Fig. 11(c) $(w=4.1 \mathrm{~mm})$. Note that the specularreflection dip in Fig. 12 overcomes the $-30 \mathrm{~dB}$ threshold at angles $19^{\circ}$ and $71^{\circ}$ (besides the $40^{\circ}$ case, corresponding to the Bragg condition). For other angles comprised between $19^{\circ}$ and $71^{\circ}$, this reflection dip also exists, although its depth is smaller. This means that, in this case, specular reflection is significantly suppressed over a wide range of incidence angles at frequencies around $8 \mathrm{GHz}$. In order to explain the existence of these two off-Bragg blazing points from the perspective of the equivalent circuit, the frequency behavior of the real and imaginary parts of the impedance $Z_{\mathrm{RG}}$ is depicted in Fig. 13 for several incidence angles. The straight horizontal lines in the plots indicate the value of the input-line impedance corresponding to the considered incidence angle $\left(Z_{0}^{(0)}\right)$. Vertical straight lines mark the resonance frequency (zero-crossing of the imaginary part). For some of the represented angles, it can be observed that the real part of $Z_{\mathrm{RG}}$ is very close to $Z_{0}^{(0)}$ at resonance (perfect matching), whereas such condition is not fulfilled for other angles. The values of the angles for which perfect matching is achieved $\left(\theta=40^{\circ}, \theta=19^{\circ}\right.$, and $\theta=71^{\circ}$ ) are the three blazing points observed in Fig. 11(c), one corresponding to Bragg blazing and two to off-Bragg blazing. 


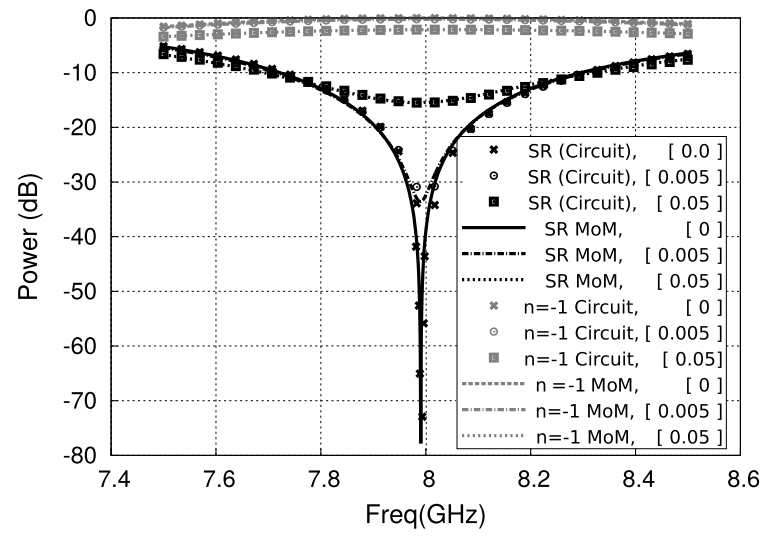

Fig. 14. Specular-reflection power (black lines) and power carried by the $n=-1$ harmonic (gray lines) for a structure with three different values $(0,0.005$, and 0.05$)$ of the loss tangent (in brackets in the inset). The dimensional parameters and the real part of the permittivity are the ones corresponding to the example in Fig. 4.

\section{EFFECT OF LOSSES}

Up to this point, the presence of material losses introduced by real dielectrics and metals has been ignored, in such a way that the proposed model would only be valid for very low-loss structures. However, as the effect of losses could be relevant in practical structures, this section is devoted to supplement the circuit model to account for losses and study their consequences. This study is particularly necessary for structures based on resonances, as it is the case in this paper, since they are very sensitive to the effect of losses [25], [26], which might completely suppress the phenomenon occurring in the absence of such losses (blazing, in our case).

\section{A. Dielectric Losses}

The inclusion of dielectric losses in the equivalent circuit is quite straightforward by simply considering, as usual, a complex permittivity describing the dielectric properties, that is

$$
\hat{\varepsilon}_{\mathrm{r}}^{(1)}=\varepsilon_{\mathrm{r}}^{(1)}(1-\mathrm{j} \tan \delta)
$$

where $\tan \delta$ is the loss tangent of the dielectric material. When this complex permittivity is used in the expressions defining the propagation constants and impedances of the transmission lines modeling the Floquet harmonics inside the dielectric slab region, these quantities all become complex at any frequency (lossy transmission lines). To have an idea of the impact of this fact on the achievable mirror-like reflection suppression, the normalized specular-reflection power for the first design reported in this article is shown in Fig. 14 for four different values of $\tan \delta$. Of course, perfect blazing is obtained in the lossless case $(\tan \delta=0)$. The reflected power curve is hardly perturbed if a typical low-loss microwave substrate ( $\tan \delta=0.005$, for instance) is considered, although perfect suppression of the specular reflection cannot be achieved at the blazing frequency (the lowest achieved reflection coefficient is about $-33 \mathrm{~dB}$ ). Nevertheless, most of the power is transferred to $n=-1$ harmonic while a minimum fraction of the power is absorbed by the substrate. For very lossy

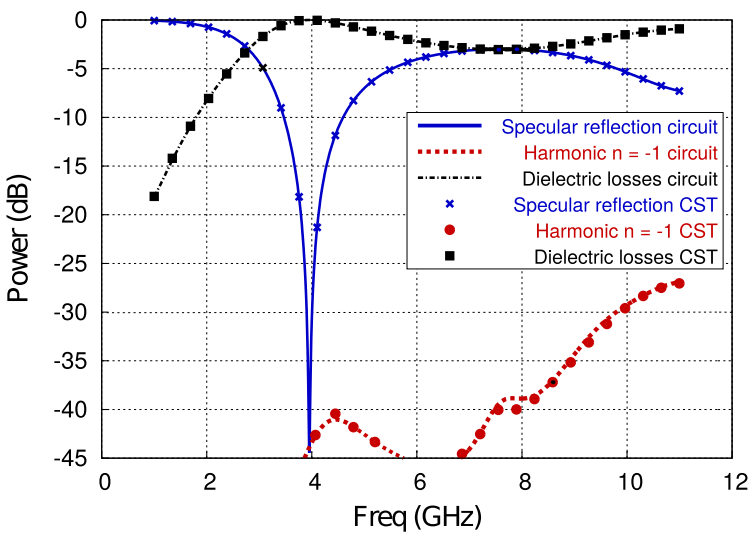

Fig. 15. Prototype of a narrow-band absorber. Structure parameters: $p=30$, $w=2.5 \mathrm{~mm}, d=6 \mathrm{~mm}, \varepsilon_{\mathrm{r}}^{(1)}=10.2, \delta=0.4, \theta=20^{\circ}$. Comparison with CST Microwave Studio [27] is included (circles, squares, and crosses).

substrates $(\tan \delta \approx 0.05$ or higher), specular reflection cannot be efficiently suppressed, which would prevent the use of this kind of substrates (for instance, FR4) for this application. From the equivalent circuit perspective, this is due to the fact that the global resistance associated with losses precludes the perfect (or quasi-perfect) matching achieved when losses are low or absent. It is worth mentioning that the agreement between the analytical (equivalent circuit) and MoM results shown in Fig. 14 is excellent, even for the highly lossy cases.

Calculations carried out with the circuit model and verified through an MoM code show that the use of a lossy substrate not only precludes good matching of the impinging wave (i.e., specular-reflection suppression) but also deviates a large portion of the impinging power to the loss mechanism rather than to the $n=-1$ harmonic. Thus, for $\tan \delta=0.05$ and $f=8.0 \mathrm{GHz}$, the relative level of power transferred to the $n=-1$ harmonic is about $-2.14 \mathrm{~dB}$ and the SR power level is $-15.51 \mathrm{~dB}$, in such a way that $36 \%$ of the impinging power is dissipated in the lossy dielectric, $2.8 \%$ is SR, and $61.2 \%$ is backscattered ( $n=-1$ harmonic). Incidentally, one might wonder if the structure can be designed in such a way that the percentage of absorbed power is close to $100 \%$ (perfect absorber). The problem of designing such kind of absorber can be posed in terms of a perfect matching problem, provided the frequency operation is below the onset of any grating lobe of the periodic structure. In such case, perfect matching means total absorption. A methodology similar to the one reported in this article for designing perfect blazing structures can also be applied to the design of a perfect absorber if very lossy substrates are used. The main difference is that all the transmission lines involved in the model are lossy. Although this is not the central subject of this article, a grating prototype aimed to yield a perfect absorber has been designed. The dimensional and electrical parameters as well as the computed electrical response are given in Fig. 15 for a TM-polarized plane wave impinging with angle $\theta=20^{\circ}$. The frequency at which perfect absorption is enforced is $4 \mathrm{GHz}$, well below the onset of the first grating lobe $(\approx 7.4 \mathrm{GHz})$. More than $90 \%$ of the impinging power is absorbed in the frequency band from 3.4 to $4.6 \mathrm{GHz}$ ( $30 \%$ fractional bandwidth). Note that 
part of the impinging power (a very small amount indeed) is transferred to the $n=-1$ harmonic at frequencies beyond 7.4 GHz. Below that cutoff frequency, the residual power transferred to this harmonic is only related to dielectric losses, of course. Comparison with full-wave results obtained using CST Microwave Studio [27] reveals very good agreement.

\section{B. Ohmic Losses}

In principle, the inclusion of ohmic losses associated with metal strips in the circuit model is not as straightforward as the inclusion of dielectric losses. However, if just an approximation to the expected level of losses is required, the concept of surface impedance of the metal regions can be used. In the formulation of the ECA, conductor strips with losses can be characterized by means of a surface impedance, $Z_{s}$, so that the resultant electric-field integral equation $[2$, eq. 30] is now

$$
\frac{1}{2} \int_{-w / 2}^{w / 2}\left[\mathbf{J}_{\mathrm{s}}\right]^{*} \mathbf{E} \mathrm{d} x=\frac{Z_{\mathrm{s}}}{2} \int_{-w / 2}^{w / 2}\left[\mathbf{J}_{\mathrm{s}}\right]^{*}\left[\mathbf{H}^{(1)}-\mathbf{H}^{(2)}\right] \mathrm{d} x .
$$

In the strong skin effect regime (the expected regime for microwave frequencies and typical thicknesses of the copper traces employed in printed circuit technology), the surface impedance is given by

$$
Z_{\mathrm{s}}=\frac{1}{\sigma \delta_{s}}(1+j)=R_{\mathrm{S}}(1+j)
$$

where $\delta_{s}$ if the skin depth. Conductor ohmic losses are then mainly controlled by the surface resistance, $R_{\mathrm{S}}$, of the metal at the operation frequency. The inclusion of the losses of the metal strips in the integral equation (29) gives rise to a slight modification of the so-called equivalent impedance $\left(Z_{\text {eq }}\right)$ defined in [23, eq. 31], which should be rewritten as (TM case)

$$
\begin{array}{r}
Z_{\mathrm{eq}}=(1+j) R_{\mathrm{s}} \sum_{\substack{n=-N \\
n \neq 0}}^{N}\left[N_{n}(\omega)\right]^{2}+\sum_{\substack{n=-N \\
n \neq 0}}^{N} \frac{\left[N_{n}(\omega)\right]^{2}}{Y_{n}^{(0)}+Y_{n, \text { in }}^{(1)}} \\
+\frac{1}{\omega C_{\mathrm{ho}}} .
\end{array}
$$

This expression coincides with the losless case in [23, eq. (31)] except for the appearance of a new resistance $R$ accounting for conductor losses:

$$
R=R_{\mathrm{S}} \sum_{\substack{n=-N \\ n \neq 0}}^{N}\left[N_{n}(\omega)\right]^{2}
$$

plus a small incremental inductance whose value (strong skin effect) is $\Delta L=R / \omega$. This complex impedance is series connected with the two boxed blocks represented in the circuit model depicted in Fig. 8. In order to check the validity of the proposed model, three lossy scenarios are analyzed in Fig. 16. The curves correspond to gratings whose strips are made of a metallic material (copper) and two different hypothetical conducting materials with relatively low values of conductivity $\left(\sigma=10^{6} \mathrm{~S} / \mathrm{m}\right.$ and $\left.\sigma=10^{5} \mathrm{~S} / \mathrm{m}\right)$ when compared with copper. The results obtained with the approximate

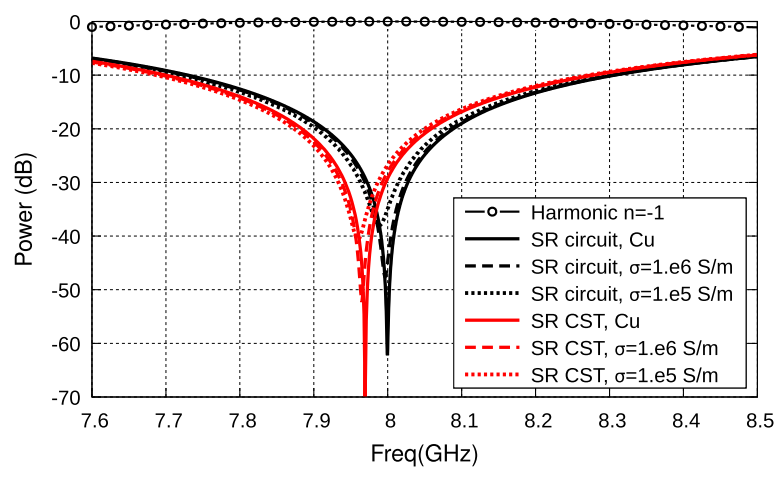

Fig. 16. SR power $(n=0)$ and power transferred to the $n=-1$ harmonic (circles) for conducting strips having three different values of surface resistance (copper, $\sigma=10^{6} \mathrm{~S} / \mathrm{m}$ and $\sigma=10^{5} \mathrm{~S} / \mathrm{m}$ ). Black curves have been computed using the equivalent circuit model and red (gray) curves have been obtained with CST Microwave Studio [27]. A single curve has been drawn for the power transferred to the $n=-1$ harmonic because the three conductivity cases visually overlap. Dimensional and electrical parameters are the ones corresponding to the example in Fig. 4.

circuit model are compared with simulation results computed using CST Microwave Studio [27], assuming a strip thickness of $35 \mu \mathrm{m}$. It can be appreciated that the approximate lossy circuit model gives rise to results close to those obtained with the computationally intensive full-wave simulations. The observed frequency shift between the two set of results (less than $0.5 \%$ difference for the resonance frequency) is partially due to the fact that the strip thickness is not accounted for in the ECA. A smaller discrepancy (less than $0.25 \%$ ) has also been observed when zero-thickness lossy strips are considered in CST simulations. In any case, the influence of ohmic losses is reasonably well captured by the model, including a tiny and subtle resonance frequency shift appearing in the highloss case $\left(\sigma=10^{5} \mathrm{~S} / \mathrm{m}\right)$. It is apparent that only very low values of the conductivity (much smaller than those typically associated with metals) would lead to significant deterioration of the blazing effect. Thus, the effect of metal losses can safely be neglected in the frame of this article.

\section{CONCLUSION}

An accurate analytical circuit model has been derived for the modeling of planar resonant blazed gratings made of periodic distributions of metal strips printed on a conductor backed substrate. Apart from accurate numerical results, the circuit model also provides a simple physical insight to explain the mechanism leading to both Bragg and off-Bragg blazing phenomena. The underlying mechanism is posed in terms of an impedance-matching condition for the transmission lines representing the impinging plane wave (zeroth order Floquet harmonic). Although perfect blazing can only be achieved at the resonance frequencies of the structure, resonance is found to be only a necessary condition. The appropriate strip width that yields perfect matching (and thus perfect cancellation of specular reflection) can be analytically obtained using the proposed circuit model. This provides a systematic method for the design of this kind of structures which does not rely on an optimization process assisted by full-wave solvers. The 
effect of material losses has also been incorporated to the circuit model. It allows us to conclude that commercially available low-loss substrates coated with copper are suitable to build satisfactory blazed gratings of this class operating at microwave frequencies.

\section{REFERENCES}

[1] M. Born and E. Wolf, Principles of Optics: Electromagnetic Theory of Propagation, Interference and Diffraction of Light, 7th ed. Cambridge, U.K.: Cambridge Univ. Press, 1999.

[2] W. C. Meecham, "Variational method for the calculation of the distribution of energy reflected from a periodic surface. I," J. Appl. Phys., vol. 27, no. 4, pp. 361-367, Apr. 1956.

[3] T. Itoh and R. Mittra, "An analytical study of the echelette grating with application to open resonators," IEEE Trans. Microw. Theory Techn., vol. 17, no. 6, pp. 319-327, Jun. 1969.

[4] A. Hessel, J. Schmoys, and D. Y. Tseng, "Bragg-angle blazing of diffraction gratings," J. Opt. Soc. Amer., vol. 65, no. 4, p. 380, Apr. 1975.

[5] J. W. Heath, Jr., and E. V. Jull, "Perfectly blazed reflection gratings with rectangular grooves," J. Opt. Soc. Amer., vol. 68, no. 9, p. 1211, Sep. 1978

[6] B. H. Kleemann, "Perfect blazing with echelle gratings in TE and TM polarization," Opt. Lett., vol. 37, no. 6, p. 1002, Mar. 2012.

[7] A. D. Squires, E. Constable, and R. A. Lewis, "3D printed terahertz diffraction gratings and lenses," J. Infr., Millim., THz. Waves, vol. 36, no. 1, pp. 72-80, Jan. 2015.

[8] S. Zhang et al., "Groove shape characteristics of echelle gratings with high diffraction efficiency," Opt. Commun., vol. 387, pp. 401-404, Mar. 2017.

[9] N. Yu et al., "Light propagation with phase discontinuities: Generalized laws of reflection and refraction," Science, vol. 334, no. 6054 pp. 333-337, Oct. 2011

[10] A. Díaz-Rubio, V. S. Asadchy, A. Elsakka, and S. A. Tretyakov, "From the generalized reflection law to the realization of perfect anomalous reflectors," Sci. Adv., vol. 3, no. 8, Aug. 2017, Art. no. e1602714.

[11] Y. Ra'di, D. L. Sounas, and A. Alù, "Metagratings: Beyond the limits of graded metasurfaces for wave front control," Phys. Rev. Lett., vol. 119 no. 6, Aug. 2017, Art. no. 067404

[12] F. Ding, A. Pors, and S. I. Bozhevolnyi, "Gradient metasurfaces: A review of fundamentals and applications," Rep. Prog. Phys., vol. 81, no. 2, Feb. 2018, Art. no. 026401.

[13] A. M. Wong and G. V. Eleftheriades, "Perfect anomalous reflection with a bipartite Huygens' metasurface," Phys. Rev. X, vol. 8, no. 1, Feb. 2018, Art. no. 011036

[14] O. Rabinovich and A. Epstein, "Analytical design of printed circuit board (PCB) metagratings for perfect anomalous reflection," IEEE Trans. Antennas Propag., vol. 66, no. 8, pp. 4086-4095, Aug. 2018.

[15] O. Rabinovich, I. Kaplon, J. Reis, and A. Epstein, "Experimental demonstration and in-depth investigation of analytically designed anomalous reflection metagratings," Phys. Rev. B, Condens. Matter, vol. 99, no. 12, Mar. 2019, Art. no. 125101.

[16] C. Molero, R. Rodriguez-Berral, F. Mesa, and F. Medina, "Analytical circuit model for 1-D periodic T-shaped corrugated surfaces," IEEE Trans. Antennas Propag., vol. 62, no. 2, pp. 794-803, Feb. 2014.

[17] X. Li, M. Memarian, K. Dhwaj, and T. Itoh, "Blazed metasurface grating: The planar equivalent of a sawtooth grating," in IEEE MTT $S$ Int. Microw. Symp. Dig., San Francisco, CA, USA, May 2016, pp. 22-27, doi: 10.1109/MWSYM.2016.7540270.

[18] M. Memarian, X. Li, and T. Itoh, "Resonant blazed metasurface gratings," in Proc. 46th Eur. Microw. Conf., London, U.K., Oct. 2016, pp. 297-300.

[19] M. Memarian, X. Li, Y. Morimoto, and T. Itoh, "Wide-band/angle blazed surfaces using multiple coupled blazing resonances," Sci. Rep., vol. 7, Feb. 2017, Art. no. 42286.

[20] L. Felsen and A. Oliner, "Determination of equivalent circuit parameters for dissipative microwave structures," Proc. IRE, vol. 42, no. 2 , pp. 477-483, Feb. 1954.

[21] A. Oliner, "Equivalent circuits for small symmetrical longitudinal apertures and obstacles," IEEE Trans. Microw. Theory Techn., vol. 8, no. 1, pp. 72-80, Jan. 1960.

[22] I. Palocz and A. Oliner, "Equivalent network of a multimode planar grating," IEEE Trans. Microw. Theory Techn., vol. 18, no. 5, pp. 244-252, May 1970.
[23] R. Rodriguez-Berral, C. Molero, F. Medina, and F. Mesa, "Analytical wideband model for strip/slit gratings loaded with dielectric slabs," IEEE Trans. Microw. Theory Techn., vol. 60, no. 12, pp. 3908-3918, Dec. 2012.

[24] C. Molero, R. Rodriguez-Berral, F. Medina, F. Mesa, M. Memarian, and T. Itoh, "Accurate circuit model for a planar resonant blazed grating," in Proc. 47th Eur. Microw. Conf. (EuMC), Nuremberg, Germany, Oct. 2017, pp. $775-778$.

[25] A. Epstein and O. Rabinovich, "Unveiling the properties of metagratings via a detailed analytical model for synthesis and analysis," Phys. Rev. Appl., vol. 8, no. 5, Nov. 2017, Art. no. 054037.

[26] V. Popov, F. Boust, and S. N. Burokur, "Controlling diffraction patterns with metagratings," Phys. Rev. Appl., vol. 10, no. 1, Jul. 2018, Art. no. 011002.

[27] (2019). CST Studio Suite. [Online]. Available: https://www.cst.com/ products/csts2

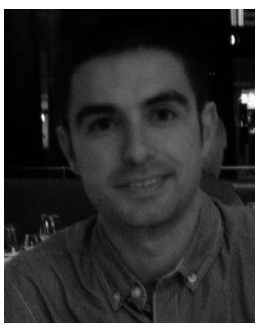

Carlos Molero was born in Seville, Spain, in April 1987. He received the Licenciado (M.Sc.) and Ph.D. degrees in physics from the Universidad de Sevilla, Seville, Spain, in 2011 and 2017, respectively.

Since March 2017, he has been a Post-Doctoral Researcher with the Institut National des Sciences Appliquées (INSA) de Rennes, Rennes, France. His research interests focus on the study of periodic structures, in planar and 3-D architectures, circuit models, full-metal devices, and design of new polarizers based on 3-D-printable self-supported cells.

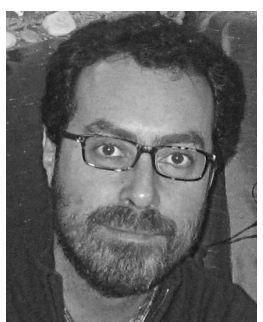

Raúl Rodríguez-Berral was born in Casariche, Seville, Spain, in 1978. He received the Licenciado (M.Sc.) and Doctor (Ph.D.) degrees in physics from the Universidad de Sevilla, Seville, Spain, in 2001 and 2008, respectively.

$\mathrm{He}$ is currently an Associate Professor with the Departamento de Física Aplicada 1, Universidad de Sevilla. His current research interest includes numerical and analytical techniques for the analysis and modeling of periodic structures.

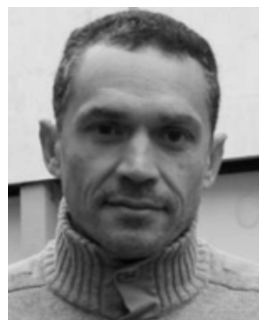

Francisco Mesa (Fellow, IEEE) received the Licenciado and Ph.D. degrees in physics from the Universidad de Sevilla, Seville, Spain, in 1989 and 1991, respectively.

He is currently a Professor with the Departamento de Física Aplicada 1, Universidad de Sevilla. His research interest includes electromagnetic propagation/radiation in planar structures. 


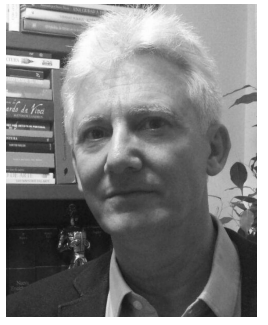

Francisco Medina (Fellow, IEEE) was born in Puerto Real, Cádiz, Spain, in 1960. He received the Licenciado (M.Sc.) and Doctor (Ph.D.) degrees in physics from the Universidad de Sevilla, Seville, Spain, in 1983 and 1987, respectively.

$\mathrm{He}$ is currently a Professor of electromagnetism with the Department of Electronics and Electromagnetism, Universidad de Sevilla, where he is also the Head of the Microwaves Group. He has co-edited a research book and published a number of book chapters, journal articles, and conference papers. His current research interests include planar passive circuits and antennas, analytical and numerical methods for planar structures, anisotropic materials, and artificial media modeling.

Dr. Medina is a member of Technical Program Committee (TPC) of the European Microwave Conference and he is in the Editorial Board of some journals in the field. He is the Editor-in-Chief of the International Journal of Microwave and Wireless Technologies

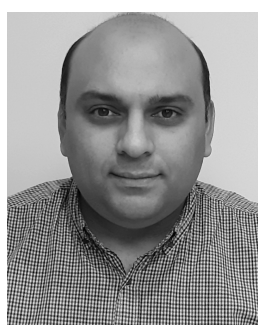

Mohammad Memarian (Senior Member, IEEE) received the B.A.Sc. degree (Hons.) in electrical engineering and the M.A.Sc. degree in electrical engineering from the University of Waterloo, Waterloo, ON, Canada, in 2007 and 2009, respectively, and the Ph.D. degree in electrical engineering from the University of Toronto, Toronto, ON, Canada, in 2015 .

He was a Post-Doctoral Fellow with the University of California at Los Angeles, Los Angeles, CA, USA. He is currently an Assistant Professor with the Sharif University of Technology, Tehran, Iran. His current research interests include electromagnetics, antennas, metamaterials and metasurfaces, periodic structures, microwave filters and system components, and electromagnetics of time-varying media.

Dr. Memarian is also a member of the National Elites Foundation of Iran. He was a recipient of various awards, including the Nortel Networks Scholarship, the Natural Sciences and Engineering Research Council of Canada (NSERC) Canada Graduate Scholarship Masters, the NSERC Post-Graduate Scholarship Doctoral, the NSERC Post-Doctoral Fellowship, the IEEE Antennas and Propagation Society Doctoral Research Award, the IEEE Microwave Theory and Techniques Society International Microwave Symposium (MTT-S IMS) Student Paper Competition Award in 2009 and 2016 (and a Finalist in 2017), the Best Oral Presentation Award of the IEEE MTT-S IMS in 2009, and the IEEE AP/URSI 2018 Student Paper Competition Award.

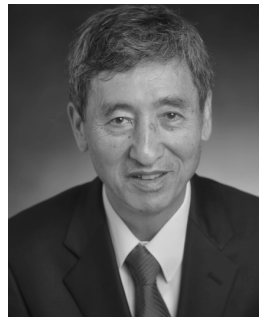

Tatsuo Itoh (Life Fellow, IEEE) received the Ph.D. degree in electrical engineering from the University of Illinois at Urbana-Champaign, Champaign, IL, USA, in 1969.

From 1976 to 1977, he was with SRI International, Menlo Park, CA, USA. From 1977 to 1978, he was an Associate Professor with the University of Kentucky, Lexington, KY, USA. In 1978, he joined The University of Texas at Austin, Austin, TX, USA, where he became the Hayden Head Centennial Professor of engineering. In 1991, he joined the University of California at Los Angeles, Los Angeles, CA, USA, as a Distinguished Professor of electrical engineering, where he holds the TRW Endowed Chair of microwave electronics (currently the Northrop Grumman Endowed Chair). He has 450 journal publications, 910 refereed conference presentations in the areas of microwaves, millimeter-waves, antennas and numerical electromagnetics. He generated $82 \mathrm{Ph} . \mathrm{D}$. students and hosted more than 100 visiting scholars and postdocs from various countries. His research has demonstrated to have technical and societal impacts on various areas not apparently related to electromagnetics. For instance, he has developed several electromagnetic modeling procedures for microwave integrated circuits which are used in the widely used smart phones. He has introduced artificial materials, such as metamaterials and bandgap structures for various wave control mechanisms beyond what was capable of while 2-D and 3-D metasurfaces appeared in use for high-power transmission and delivery. Millimeter and $\mathrm{THz}$ applications have been found efficient if the passive and active co-design is carefully done based on rigorous and fundamental electromagnetic theory. At high frequencies, the role of the integrated antennas containing more than radiating mechanisms becomes non-negligible and often the key to success.

Dr. Itoh was elected as an Honorary Life Member of the MTT Society in 1994 and elected to a member of the National Academy of Engineering in 2003. In 2014, he was inducted to the National Academy of Inventors as a fellow. He was given fellow status of the International Union of Radio Science (URSI) in 2017. He received several awards, including the IEEE Third Millennium Medal in 2000, the IEEE MTT Distinguished Educator Award in 2000, the Outstanding Career Award from the European Microwave Association in 2009, the Microwave Career Award from the IEEE MTT Society in 2011, the Alumni Award for his distinguished service from the College of Engineering, University of Illinois at Urbana-Champaign, in 2012, and the IEEE Electromagnetics Award in 2018. He also received the Doctor Honoris Causa, Universitat Autònoma de Barcelona, Spain, in October 2014. $\mathrm{He}$ is listed as the number one author in electrical engineering by the Microsoft Academic Search in 2014. He was the Editor of the IEEE TRANSACTIONS on Microwave Theory AND Techniques from 1983 to 1985 and the founding Editor-in-Chief of the IEEE MiCROWAVE AND GUIDED WAVE LETTERS from 1991 to 1994 . He was the President of the MTT Society in 1990. He served as a Distinguished Microwave Lecturer of MTT-S from 2004 to 2006. He was the Chairman of the Commission D of URSI from 1993 to 1996. 